\title{
Effects of the Micro-hole Target Structures on the Laser- driven Energetic Proton Generation
}

\author{
Ki Hong Pae ${ }^{1}$, II Woo Choi ${ }^{1}$, Sang June Hahn ${ }^{2}$, and Jongmin Lee ${ }^{1 *}$ \\ ${ }^{1}$ Advanced Photonics Research Institute \& Center for Femto-Atto Science and Technology, \\ GIST, Gwangju 500-712, Korea \\ ${ }^{2}$ Department of Physics, Chung-Ang University, Seoul 156-756, Korea
}

(Received January 5, 2009 : revised Feburary 2, 2009 : accepted March 4, 2009)

\begin{abstract}
Micro-hole targets are studied to generate energetic protons from laser-thin foil targets by using 2-dimensional particle-in-cell simulations. By using a small hole, the maximum energy of the accelerated proton is increased to 4 times higher than that from a simple planar target. The main proton acceleration mechanism of the hole-targets is the electrostatic field created between the fast electrons accelerated by the laser pulse ponderomotive force combined with the vacuum heating and the target rear surface. But in this case, the proton angular distribution shows double-peak shape, which means poor collimation and low current density. By using a small cone-shaped hole, the maximum proton energy is increased 3 times higher than that from a simple planar target. Furthermore, the angular distribution of the accelerated protons shows good collimation.
\end{abstract}

Keywords : PIC simulation, Laser-plasma, Proton acceleration, Micro-hole target

OCIS codes: (140.3460) Lasers; (350.5400) Plasmas

\section{INTRODUCTION}

Energetic protons accelerated by short pulse laserthin foil interactions have promising properties such as laminarity, short duration and good collimation. The target normal sheath acceleration (TNSA) mechanism has been studied extensively both theoretically $[1,2]$ and experimentally $[3-5]$ as a basic mechanism for the generation of laser-driven energetic protons. Effects of preplasma and target density profile on the proton energy also have been investigated $[6,7]$. Also, several ideas on target design to generate high-quality, high-efficiency and high-energy protons have been proposed. Flippo et al used flat-top cone targets to get high-efficiency proton beams [8]. By using particle-in-cell (PIC) simulations, Liu et al showed that energetic collimated ion bunches can be achieved with a thin concave target [9]. A micro-structured double layer target was proposed for the generation of high-quality ion beams [10]. Cowan et al successfully demonstrated a laser virtual-cathode method to get an ultralow-emittance energetic proton beam [11]. Pfotenhauer et al used microstructured targets

*Corresponding author: leejm@gist.ac.kr to reliably produce monoenergetic protons[12].

In this work, we use 2-dimensional PIC simulations to study the effects of the micro-hole structures on the thin double-layer target for the generation of energetic protons driven by an intense, ultrashort laser pulses..

\section{SIMULATION SCHEME}

We use VORPAL [14] code to study the proton acceleration in laser-thin foil target interactions. It is a well known fully relativistic electromagnetic PIC code. The schematics of the simulation are shown in Fig. 1. A thin foil target (thickness $l_{0}=5 \mu \mathrm{m}$ ) is located at $\mathrm{x}=2.5 \mu \mathrm{m}$. The target is assumed to be fully ionized by laser prepulse and amplified spontaneous emission (ASE). The target is modeled by $\mathrm{Cu}$ ions and electrons with density $\mathrm{n}_{\mathrm{e}}=\mathrm{n}_{0}=2 \times 10^{22} / \mathrm{cm}^{3}$. The contamination layer is composed of protons and electrons with density $\mathrm{n}_{\mathrm{p}}=\mathrm{n}_{\mathrm{e}}=\mathrm{n}_{1}=1 \times 10^{21} / \mathrm{cm}^{3}$ and thickness $l_{1}=25 \mathrm{~nm}$. An intense $\left(I=1 \times 10^{19} \mathrm{~W} / \mathrm{cm}^{2}\right)$, ultrashort $\left(\tau_{\mathrm{L}}=34 \mathrm{fs}\right.$ at full-width half-maximum $)$, tightly focused ( $\omega_{0}=6 \mu \mathrm{m}$ at full-width half-maximum), 
p-polarized laser pulse is injected from the left side of the simulation window and is focused on the front surface of the foil target. The laser pulse has Gaussian profiles in both spatial and temporal coordinates, and its wavelength is $0.8 \mu \mathrm{m}$. In this case, the Rayleigh range is about $140 \mu \mathrm{m}$ so that the laser pulse holds almost constant intensity within the target.

The total simulation window size is $192 \mu \mathrm{m} \times 128 \mu \mathrm{m}$ and the grid cell size is $25 \mathrm{~nm} \times 25 \mathrm{~nm}$ so that the total number of gird is $3.9 \times 10^{7}$. The contamination layer is represented by 128 macro-particles per grid cell.

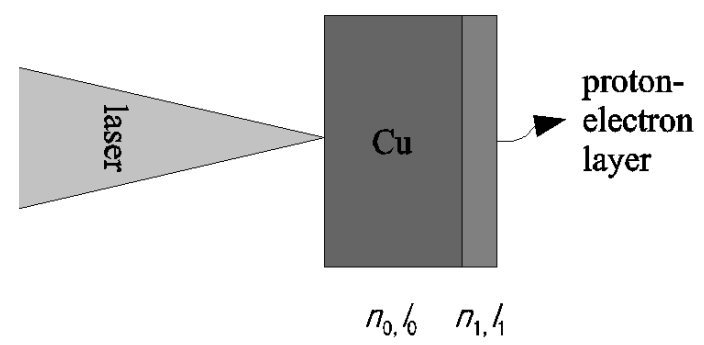

FIG. 1. Simulation scheme.

\section{SIMULATION RESULTS}

The typical simulation result of short-pulse laserdouble layer target interaction is given in Fig. 2. Figure $2(\mathrm{a})$ is the proton phase space, 2(b) is the energy spectrum of the protons, and 2(c) is the angular distribution of the accelerated protons. These results show characteristics of the TNSA model. In many laser-based proton acceleration experiments using thin foil targets, double-layer structures are created by contamination of hydrocarbon and/or water vapor residing on the target rear surface. For a double-layer target, the proton acceleration by short pulse laser is well explained by the TNSA model. In the TNSA model, the sheath field created by the fast electrons accelerated by laser ponderomotive potential accelerates protons originating at the target back surface. Because the sheath field is normal to the target surface, the TNSA mechanism results in a high current density and well-collimated protons. Furthermore, the accelerated ion front has a sharp boundary with vacuum, which results in a sharp cut-off energy. The shielding effect of the sheath field by accelerating ions results in extremely laminar ion acceleration. The maximum energy of the accelerated proton from

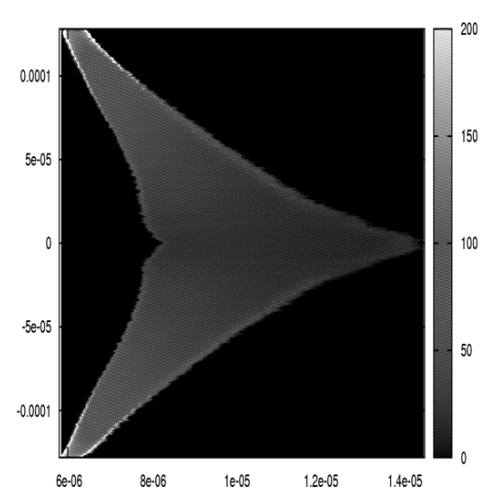

(a)

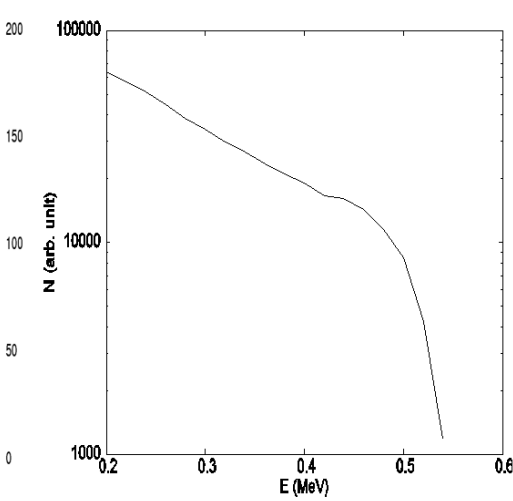

(b)

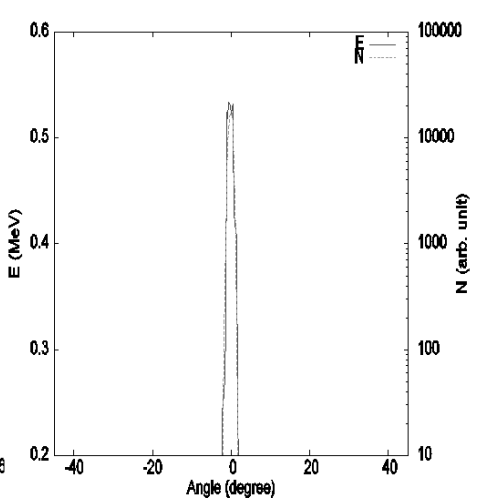

(c)

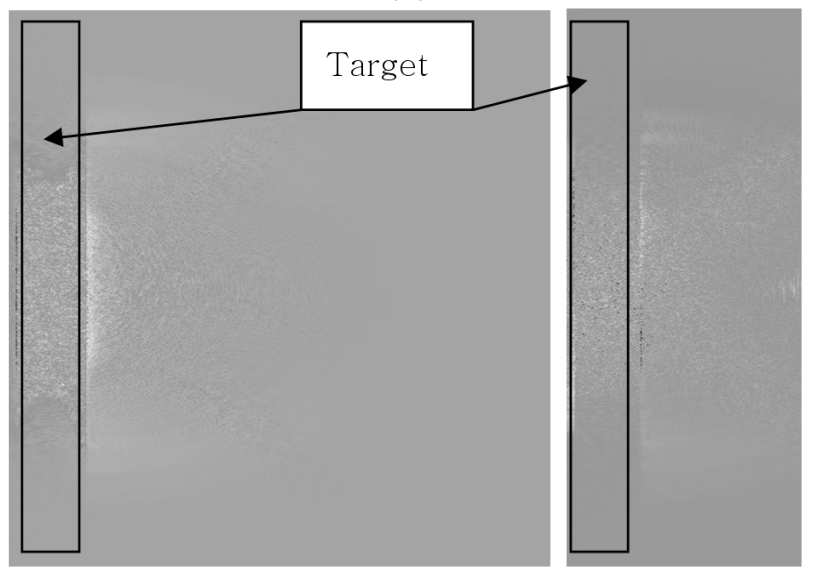

(d)

(e)

FIG. 2. (a) Proton phase space, (b) energy spectrum of the protons, and (c) angular distribution of the accelerated protons (E : energy, N : number). (d) The x-component of the electric field and (e) z-component of the magnetic field. 
a double-layer target is given as $\varepsilon_{\max }=2 \varepsilon_{0}(\ln (2 \tau))^{2}$ according to the TNSA model, which is determined by the target density and the laser intensity and the laser pulse duration[13]. Here, $\varepsilon_{0}=\mathrm{Zk}_{\mathrm{b}} \mathrm{T}_{\mathrm{e}}, \tau=\omega_{\mathrm{pi}} \mathrm{t}_{\mathrm{acc}} /(2 e)^{0.5}$, $\mathrm{Z}$ is the ion charge number, $\mathrm{k}_{\mathrm{b}}$ is the Boltzmann constant, $\omega_{\mathrm{pi}}$ is the ion plasma frequency, $\mathrm{t}_{\mathrm{acc}}$ is the acceleration time, and $e$ is the Euler number. Figure 2(a) shows the laminarity of the accelerated protons. In Figure

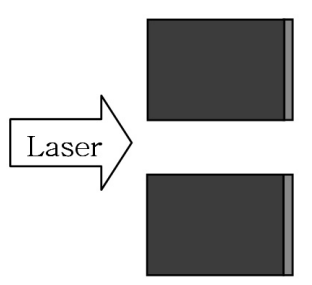

(a)

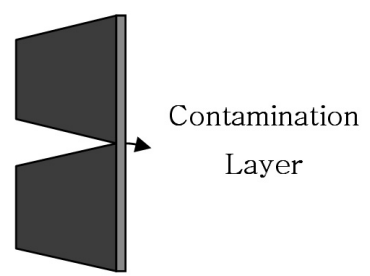

(b)
FIG. 3. (a) Simple hole on the thin-foil target. (b) Cone-shaped hole on the thin-foil target. 2(b), the sharp cut-off of the proton energy spectrum is clearly seen. However, the maximum proton energy from the simple double-layer target is slightly lower than that calculated by a simple analytical model because of the ultrashort laser pulse duration. Figure 2(c) shows the well-collimated property of the accelerated protons. In Figure 2(d) and Figure 2(e), electric field and magnetic field are shown. The strong electric field at the target rear surface accelerates protons and the magnetic field created by the fast electrons confines high energy protons in a small angle.

To see effects of micro-hole structures in thin-foil targets, we examined a hole target and a cone-shapedhole target as shown in Figure 3. The hole diameter is $2 \mu \mathrm{m}$. These structures are designed to couple the laser ponderomotive force and the vacuum-heated electrons[15] effectively.

The simulation results are shown in Figure 4. In Figure 4(a), the maximum proton energy is enhanced more than 4 times more than that from the simple

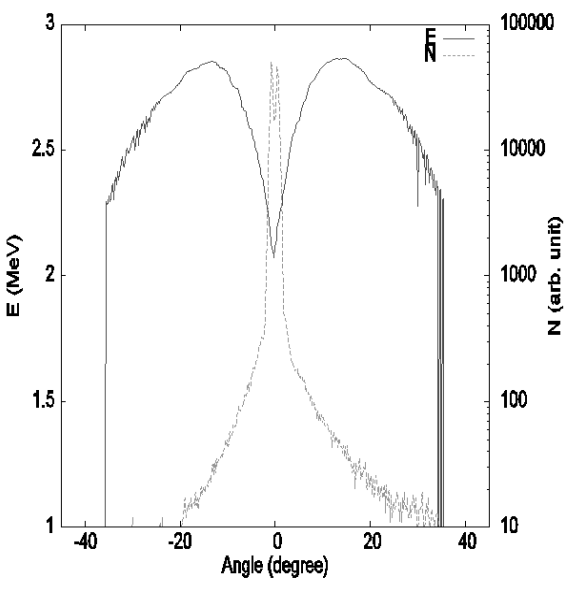

(a)

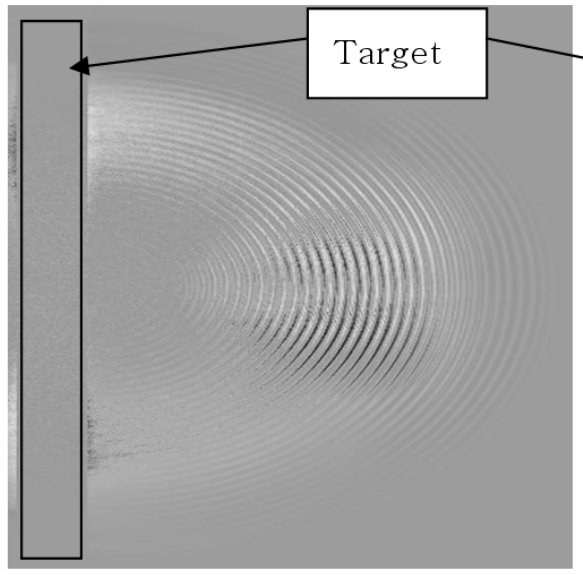

(c)

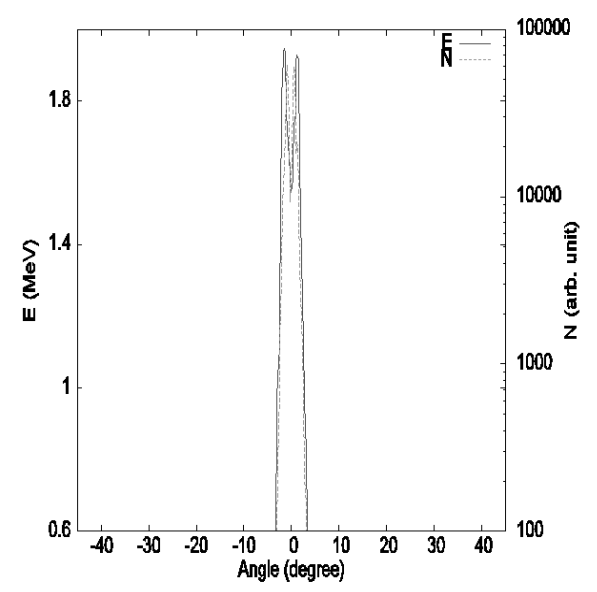

(b)

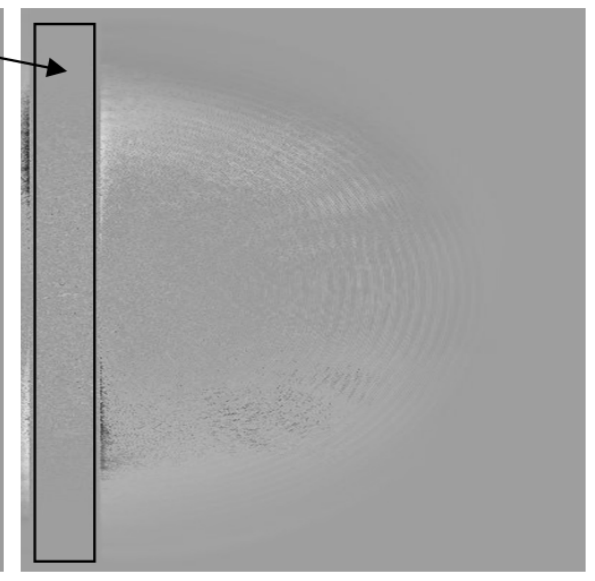

(d)

FIG. 4. Fig. 4. Angular distribution spectrum of the accelerated protons (a) from the simple hole target, (b) from the cone-shaped hole target (E : energy, $\mathrm{N}$ : number). The z-components of magnetic field (c) for the simple hole target and (d) for the cone-shaped hole target. 
plain target. But the angular distribution shows large divergence. The basic proton acceleration process is understood as follows:

While the laser pulse propagates through the target hole, electrons in the target are heated by a vacuum heating mechanism and accelerated into the hole region. These electrons are accelerated by the laser pulse ponderomotive force in the longitudinal direction inside the hole. When the laser pulse passes the rear surface of the target, its spatial profile diverges in the transverse direction. The transverse component of the laser pulse ponderomotive force acts on the electrons so that the electrons are accelerated rapidly in the transverse direction. These fast electrons accelerate the protons by the electrostatic field created between the fast electrons and the target rear surface. Also, two branches of strong magnetic field are generated along the fast electron path as shown in Figure 4(c). This results in the low current density of the accelerated protons. This process results in the double peak picture in the angular distribution spectrum as can be seen in Figure 4(a).

From the Figure 4(b) we see a well-collimated angular distribution similar to that from a simple plain target (Figure 2(c)). This is followed by the confining magnetic field as shown in Figure 4(d). Furthermore, the maximum proton energy is about 3 times higher than that from a simple plain target. The vacuum-heated electrons are accelerated normal to the vacuum-plasma boundary so that the longitudinal acceleration is smaller than that of a hole target. The laser pulse ponderomotive force is blocked at the target rear surface so that the transverse angular spread as in Fig. 4(a) does not occur. This analysis explains the result well.

In Figure 5, the energy spectra are compared for all three target types. Note the two-temperature structure on the proton energy spectrum for the hole target and

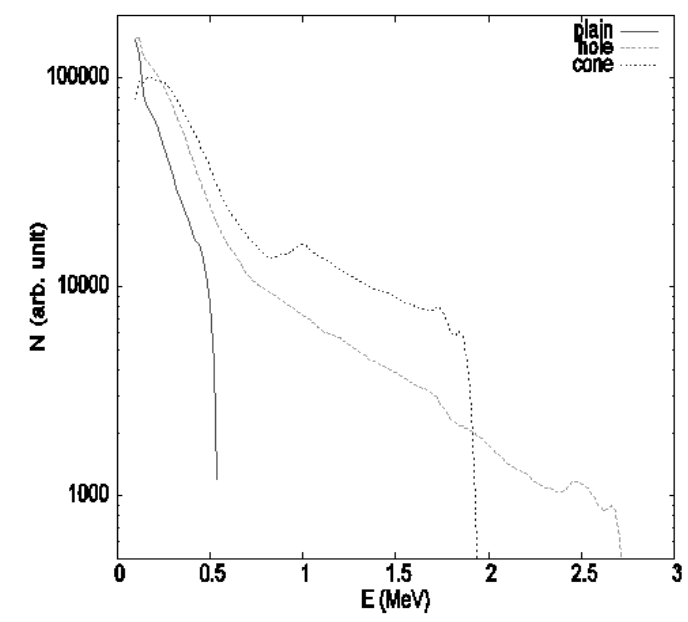

FIG. 5. Comparison of the proton energy spectrum of different target types. the cone-shaped hole target. This structure is not clear for the simple planar target case.

\section{CONCLUSION}

In conclusion, we have studied the effects of target micro-hole-structures on the proton acceleration driven by an ultrashort, intense laser pulse. By using a holetarget, the maximum proton energy is enhanced by 4 times. The laser pulse ponderomotive force on the electrons is exerted efficiently to produce high-energy electrons. In the simple hole target case, the resulting accelerated protons show large transverse divergence caused by laser pulse transverse ponderomotive force behind the target rear surface. A cone-shaped-hole removed the transverse distribution problem by stopping the laser pulse at the target rear surface. The maximum proton energy from the cone-shaped-hole target is about 3 times higher than that from the plain doublelayer target without the emittance growth.

\section{ACKNOWLEDGMENT}

This work was partially supported in part by the Ministry of Knowledge and Economy of Korea through the Ultrashort Quantum Beam Facility Program, and also by the Korea Foundation for International Cooperation of Science \& Technology (KICOS) through a grant provided by the Ministry of Education, Science and Technology of Korea (No. K20724000002-07B0400-00210).

\section{REFERENCES}

1. S. C. Wilks, A. B. Langdon, T. E. Cowan, M. Roth, M. Singh, S. Hatchett, M. H. Key, D. Pennington, A. Mackinonn, and R. A. Snavely, "Energetic proton generation in ultra-intense laser-solid interactions," Phys. Plasmas 8, 542-549 (2001).

2. A. Macchi, F. Cattani, T. V. Liseykina, and F. Cornolti, "Laser acceleration of ion bunches at the front surface of overdense plasmas,” Phys. Rev. Lett. 94, 165003 (2005).

3. S. P. Hatchett et al, "Electron, photon, and ion beams from the relativistic interaction of petawatt laser pulses with solid targets," Phys. Plasmas 7, 2076-82 (2000).

4. A. Maksimchuk, S. Gu, K. Flippo, D. Umstadter, and V. Yu. Bychenkov, "Forward ion acceleration in thin films driven by a high-intensity laser,” Phys. Rev. Lett. 84, 4108-11 (2000).

5. M. Allen, P. K. Patel, A. Mackinnon, D. Price, S. Wilks, and E. Morse, "Direct experimental evidence of back-surface ion acceleration in laser-plasma interactions," Phys. Rev. Lett. 93, 265004 (2004).

6. H. J. Lee, K. H. Pae, H. Suk, and S. J. Hahn, "Enhancement of high-energy ion generation by preplasmas in the inter- 
action of an intense laser pulse with overdense plasmas," Phys. Plasmas 11, 1726-9 (2004).

7. A. J. Mackinnon, M. Borghesi, S. Hatchett, M. H. Key, P. K. Patel, H. Campbell, A. Schiavi, R. Snavely, S. C. Wilks, and O. Willi, "Effect of plasma scale length on multi-MeV proton production by intense laser pulses," Phys. Rev. Lett. 86, 1769-72 (2001).

8. K. A. Flippo et al, "Increased efficiency of short-pulse laser-generated proton beams from novel flat-top cone targets," Phys. Plasmas 15, 056709 (2008).

9. M. P. Liu, H. C. Wu, B. S. Xie, J. Liu, H. Y. Wang, and M. Y. Yu, "Energetic collimated ion bunch generation from an ultraintense laser interacting with thin concave targets," Phys. Plasmas 15, 063104 (2008).
10. T. Esirkepov et al, "Proposed double-layer target for the generation of high-quality laser-accelerated ion beams," Phys. Rev. Lett. 89, 175003 (2002).

11. T. E. Cowan et al, "Ultralow emittance, multi-MeV proton beams from a laser virtual-cathode plasma accelerator,” Phys. Rev. Lett. 92, 204801 (2004).

12. S. M. Pfotenhauer et al, "Spectral shaping of laser generated proton beams," New J. Phys. 10, 033034 (2008).

13. P. Mora, "Plasma expansion into a vacuum," Phys. Rev. Lett. 90, 185002 (2003).

14. C. Nieter and J. R. Carry, "VORPAL: a versatile plasma simulation code,” J. Comput. Phys. 196, 448-73 (2004).

15. F. Brunel, "Not-so-resonant, resonant absorption," Phys. Rev. Lett. 59, 52-5 (1987). 